\title{
correspondence
}

\section{Low-level radiation}

StR,-Several times in the last two years and particularly during the Windscale hearings it has been suggested that the linear hypothesis for production of cancer or mutations by radiation could not be relied upon at very low levels of dose. It has, of course, for some time been recognised that there is and can be no actual evidence for damage to humans by very low levels of dose since the effects to be expected are so common from other unknown causes that there would be no hope of distinguishing a few extra cases resulting from low-level doses even if these were to affect large populations. There could thus be a threshold dose below which the effects were zero. This seems unlikely on theoretical grounds and it is obviously wise to play safe by assuming that there is no such threshold.

The suggestion with which I am concerned, however, is that very low levels of dose might have effects which are actually larger than those to be expected from the linear hypothesis. This seems to be highly improbable for the following reasons:

It is generally agreed from the near linearity with dose of the incidence of malignant disease due to higher levels of radiation that the primary effect of radiation is to produce a permanent change in a single cell. The cell is thereby either caused later on to undergo uncontrolled proliferation or alternatively is left in a state in which it can be induced to do so by some common external cause occurring later in the life of the individual concerned. At the dose rates with which I am concerned (below $100 \mathrm{mrems}$ ) any non-linear theory would require some very improbable assumptions.

I will consider first the alpha-emitting radioactive materials, which are most dangerous and which are perhaps causing the greatest concern owing to their long half-lives. If a single alpha particle dissipated its energy in a single cell (mass perhaps $3 \times 10^{-8}$ of a gramme) it would give a radiation dose to that cell of about 25 rems (assuming a quality factor of 10). Since the range of one of the alpha particles would be a little more than the diameter of a typical cell one can assume that in a large proportion of cases the alpha energy will be divided between 2 or 3 cells so that the average dose to each cell affected would be in the region of 10 rems.

If then a person is receiving a local dose of 100 mrems per year due to alpha-emitters, an individual cell can expect to the struck by an alpha particle once in 100 years. The number of cells struck once is of course proportional to dose, and this implies exceedingly strongly that any effects in this dose range must vary linearly with dose. If smaller dose rates still had a larger than proportional effect, a second event, happening decades later, would have to be able to cure the effect of an early event. At high dose dose rates this could be true since there is a fair chance that a second alpha particle would kill a previously affected cell outright, but it does not seem sensible to suppose that individual cells can be cured of whatever damage has been incurred by further radiation damage, many years later, either in the cell itself let alone in one of the surrounding cells.

The same argument applies to beta and gamma radiation although here for a given number of mrems per year the number of cells receiving a significant energy input is perhaps 10 or 20 times larger. In this case, however, since everyone in this country has a background dose of 100 mrems or more, what we would have to believe is that although this first dose between perhaps 100 to 200 mrems has an unobservably small effect, an additional dose of a few mrems anywhere in this range may have a disproportionately serious effect. Your faithfully

Department of Physics JOHN FREMLIN
University of Birmingham

\section{Pit latrines}

SIR,-In many places the best way of providing sanitation for the millions in developing countries who live in rural areas and makeshift city accommodation is still the pit latrine. At Loughborough a team is investigating the ubiquitous privy along with other forms of unsewered sanitation. Unfortunately there are precious few facts and figures to work on.

In the days of the Empire, latrine programmes often fell to the lot of government agents, district commissioners and other administrators who were renowned for scrupulous note-taking. I wonder, Sir, whether any of your readers have retained notes or have a clear memory of the operation of pit latrines. If so, will they please get in touch with me, especially if they have information about such things as the life of the pit, groundwater pollution, successful modification to prevent nuisance, and use as fertiliser of the mature contents of pits.

$$
\text { Your faithfully }
$$

University of Technology OHN PICKFORD Loughborough

\section{Nature and nurture}

SIR,-In recent years a great deal of heat and little if any light has been generated by discussions of the inheritability or otherwise of 'intelligence' (whatever this term may mean), especially as applied to supposedly genetically isolated ethnic groupings. To my knowledge, no attempt has been made to examine whether there are any simpler or more readily defined characteristics than 'intelligence' which could illuminate whether there was any scientific point whatever in such investigations. This note attempts to help in this respect.

Few would deny that physical attributes are less complex than mental attributes. Furthermore, there exists a hierarchy of complexity in human physical attributes. Some (for example, colour of eyes or hair, colour blindness, and haemophilia) are easily defined, readily measured, constant throughout life, and it seems to be reasonable to classify them as 'simple'. Next one might take measures like bodyweight or height or size of biceps or girth at various ages, characteristics that might be called 'intermediate' in this classification. The highest forms of athletic skills (pole vaulting, running, pentathlon etc) are however without doubt highly complex.

There is little doubt that the 'simple' attributes are genetically determined, as is established by massive investigations, nor is there much debate that both genetic and environmental factors (such as nutrition) are important for the 'intermediate' characteristics.

As regards the highly complex athletic skills, they are measured in various forms of international competitions, notably the Olympic Games. Due to the political circumstances of the post World War Two period, an illuminating amount of information has become available.

Before 1945, the genetic differences between the areas that became East and West Germany respectively were quite modest, certainly by comparison with the differences between major ethnic groupings. After 1945, there was first a mass movement of Germans from further east, largely to West Germany, and then, until 1961, a substantial movement of people from East Germany into West Germany. The first of these would have diminished yet further the genetic difference between the two, as would the second (which, some would say, had impoverished the genetic pool of East Germany).

Since the DDR (East Germany) has participated in international sporting contests, the outstanding performance of their athletes has excited widespread comment, particularly when the modest size of the population of the DDR is remembered. There is thus little doubt that the great support given to sport (and athletics in particular) by the DDR has enabled the country to put up a remarkable performance relative to the bigger and richer West Germany. Since this success of the DDR can hardly be ascribed to genetic differences vis a vis West Germany, it follows unambiguously that environment must have a commanding part to play in establishing complex human physical achievements, in spite of the undoubtedly genetic determination of simple physical characteristics, such as eve colour.

With such evidence plainly in front of us, what scientific sense can be assigned to attempts to establish the genetic basis of such incredibly complex attributes as 'intelligence'?

\section{Yours faithfully}

HERMANN BONdi

Department of Energy

London 